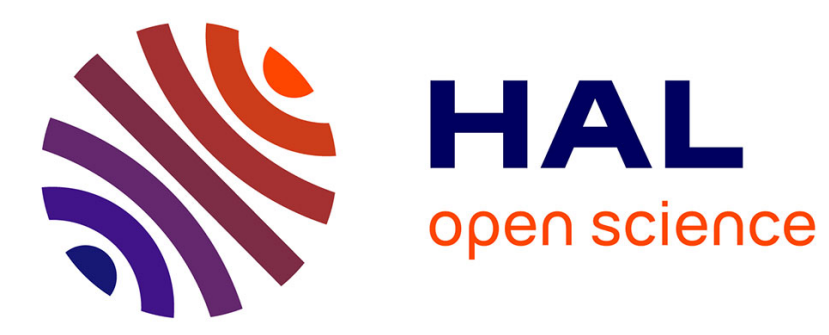

\title{
Assimilation of spatially distributed water levels into a shallow-water flood model. Part I: Mathematical method and test case
}

Xijun Lai, Jerome Monnier

\section{- To cite this version:}

Xijun Lai, Jerome Monnier. Assimilation of spatially distributed water levels into a shallow-water flood model. Part I: Mathematical method and test case. Journal of Hydrology, 2009, 377, pp.1-11. hal-00908258

\section{HAL Id: hal-00908258 \\ https://hal.science/hal-00908258}

Submitted on 22 Nov 2013

HAL is a multi-disciplinary open access archive for the deposit and dissemination of scientific research documents, whether they are published or not. The documents may come from teaching and research institutions in France or abroad, or from public or private research centers.
L'archive ouverte pluridisciplinaire HAL, est destinée au dépôt et à la diffusion de documents scientifiques de niveau recherche, publiés ou non, émanant des établissements d'enseignement et de recherche français ou étrangers, des laboratoires publics ou privés. 


\title{
Assimilation of spatially distributed water levels into a shallow-water flood model, Part I: mathematical method and test case
}

\author{
X. Lai, J. Monnier \\ see the publication in J. Hydrology
}

\begin{abstract}
Recent applications of remote sensing techniques produce rich spatially distributed observations for flood monitoring. In order to improve numerical flood prediction, we have developed a variational data assimilation method (4D-var) that combines remote sensing data (spatially distributed water levels extracted from spatial images) and a $2 \mathrm{D}$ shallow water model. In the present paper (part I), we demonstrate the efficiency of the method with a test case. First, we assimilated a single fully observed water level image to identify time-independent parameters (e.g. Manning coefficients and initial conditions) and time-dependent parameters (e.g. inflow). Second, we combined incomplete observations (a time series of water elevations at certain points and one partial image). This last configuration was very similar to the real case we analyze in a forthcoming paper (Part II). In addition, a temporal strategy with time overlapping is suggested to decrease the amount of memory required for long-duration simulation.
\end{abstract}

\section{Introduction}

Numerical flood models based on two-dimensional shallow water equations (StVenant equations) are widely used to analyze flood events or the effects of flood control projects. Computational codes that numerically simulate such surface flows are becoming more and more efficient and accurate. Nevertheless, in order to run reliable numerical simulations, one must calibrate the model parameters and know the boundary conditions (e.g. incoming discharge) and initial conditions. In river hydraulics, the parameter most often calibrated is the Manning roughness coefficient. Typically, one uses the trial and error method to calibrate this spatially distributed parameter. Available observations are only used to validate the numerical results a posteriori. Calibration "by hand" can be time-consuming and successful simulation depends strongly on expert experiences in most cases. 
The variational data assimilation method (4D-var) based on the optimal control theory of partial differential equations is a powerful tool for optimally fusing measurements and the mathematical model. On the one hand, this method allows for sensitivity analysis, which can greatly help the expert-user to calibrate "by hand" (computation of a gradient). On the other hand, it offers an optimal way of identifying some input parameter values using an optimization procedure. The optimization procedure minimizes the discrepancy between data and the model response. This approach, based on the derivation of an adjoint model, has proven its efficiency in operational meterology (see [17],[4] for pioneering articles), and more recently in operational oceanography.

In river hydraulics, variational data assimilation methods have been used successfully for one-dimensional and two-dimensional shallow water equations in $[1,5,2,23,3,11,7,12,13]$.

Also, models have been calibrated using the GLUE method [16] or, in the case of one-dimensional equations, Kalman filtering approach [9, 20].

More and more observation data are now available, especially with modern remote sensing techniques. The full potential of heterogeneous observations has not yet been reached. If good corresponding observation operators are defined, then variational data assimilation methods can fuse heterogeneous observations and the mathematical model.

In this paper, we show the potential utility of the variational data assimilation method in river hydraulics if spatially distributed water levels are available. Such observations can be extracted from satellite images [15, 22]. We show how the images can help identify some unknown input parameters and optimally fit the computed flow and observed water levels. The use of a remote sensing image for a Moselle river (France) flood event is described in a forthcoming article (Part II).

The paper is organized as follows. Section 2 describes the flow model: two-dimensional shallow water equations. The adjoint model, the full optimal control process and its implementation are presented in Section 3. Section 4 presents a test case and a definition of the total cost function for assimilation of spatially distributed water levels. In Section 5 , special attention is given to an extra penalty term in the cost function and the improvement of the identification process. In Section 6, we describe the identification of initial conditions or inflow discharge from "full images", i.e. images that provide water levels everywhere in the domain at a single instant. In Section 7, we address the problem of the identification of inflow discharge when only one partial image is available, along with pointwise measurements of water level from a gauging station in the main channel. Finally, because long-term simulations can be hindered by limited memory, we present in Section 8 a temporal strategy that includes overlapping to identify time-dependent parameters with a lesser memory requirement. 


\section{Two-dimensional flood model}

\subsection{Basic equations}

Shallow water equations(2D-SWEs) effectively model the flooding process in a floodplain or wetland. Their conservative form is (see e.g. [26]):

$$
\frac{\partial \mathbf{U}}{\partial t}+\frac{\partial \mathbf{F}(\mathbf{U})}{\partial x}+\frac{\partial \mathbf{G}(\mathbf{U})}{\partial y}=\mathbf{B}(\mathbf{U})
$$

where $x$ and $y$ are the space coordinates, $t$ is time, $\mathbf{U}$ is the state of the flow, $\mathbf{F}$ and $\mathbf{G}$ are the $x$ - and $y$-directional flux vectors and $\mathbf{B}$ is the source-term vector. These vectors are defined as follows:

$$
\begin{array}{r}
\mathbf{U}=(h, h u, h v)^{T}=\left(h, q_{x}, q_{y}\right)^{T} \\
\mathbf{F}=\left(h u, h u^{2}+\frac{1}{2} g h^{2}, h u v\right)^{T} \\
\mathbf{G}=\left(h v, h u v, h v^{2}+\frac{1}{2} g h^{2}\right)^{T} \\
\mathbf{B}=\left(0, g h\left(S_{0 x}-S_{f x}\right), g h\left(S_{0 y}-S_{f y}\right)^{T}\right.
\end{array}
$$

where $h$ is the water depth, $u$ and $v$ are the velocity components, $q_{x}=h u$ and $q_{y}=h v$ are the unit discharge components, $S_{0 x}$ and $S_{f x}$ are the bed and friction slopes respectively along $x$, and similarly $S_{0 y}$ and $S_{f y}$ along $y$. The friction slopes are evaluated using the Manning formula:

$$
S_{f x}=\frac{n^{2} q_{x} \sqrt{\left(q_{x}\right)^{2}+\left(q_{y}\right)^{2}}}{h^{7 / 3}}, \quad S_{f y}=\frac{n^{2} q_{y} \sqrt{\left(q_{x}\right)^{2}+\left(q_{y}\right)^{2}}}{h^{7 / 3}}
$$

where $n$ is the Manning coefficient.

To simulate the flood event with 2D-SWEs, initial conditions and boundary condition must be defined. Initial conditions are:

$$
\mathbf{U}_{0}=\left(\left.h\right|_{t=t_{0}},\left.q_{x}\right|_{t=t_{0}},\left.q_{y}\right|_{t=t_{0}}\right)^{T}
$$

The boundary conditions are as follows:

- At upstream boundaries, the normal discharge $q_{n}(t)$ and the normal gradient of elevation $\frac{\partial h}{\partial x_{n}}$ are imposed,

- At open downstream boundaries, elevation $h(t)$ is imposed,

- At land (wall or closed) boundaries, the normal discharge and the normal gradient of elevation are imposed to vanish:

$$
q_{n}=0 \text { and } \frac{\partial h}{\partial x_{n}}=0
$$




\section{$2.2 \quad$ Numerical method}

The 2D-SWEs (1)-(8) are solved using DassFlow software, [11]. The numerical scheme is a finite volume scheme, implemented on an unstructured mesh (triangles and/or quadrilaterals). The solver uses the rotational invariant property of the equations, then it uses a local 1D HLLC Riemman solver [26] with a consistent intermediate wave speed, [6]. It has first-order accuracy in space but a high resolution for flow discontinuity. Time discretization is done using the forward Euler scheme so a stability condition must be respected: $\Delta t \leq \frac{\min \left(d_{L, R}\right)}{\max \left(\left|u_{n}\right|+c\right)}$, where $d_{L, R}$ is the distance between two cell centerse and $u_{n}$ is the normal vector at interface.

The friction source term in momentum equations is semi-implicit in time [11].

Wetting and drying process are taken into account as follows, see e.g. [26] and [27]. Within the framework of unstructured finite volume methods, one very small water depth denoted by $h_{\epsilon}$ is considered the tolerance for determining the dynamic boundary of a cell interface in the flood front, across which the normal fluxes are calculated using corresponding methods. The values of water depth $h_{L}, h_{R}$ on both sides of the calculated cell interface are checked at each time step. There are three possibilities:

- If both values are zero (in this study, water depths less than $h_{\epsilon}=10^{-4} \mathrm{~m}$ are considered zero), we consider the cell dry and zero mass and momentum fluxes are simulated.

- If only one of them is zero, the normal fluxes are calculated with an analytic solution (formula of weir equation, see e.g. [27] p. 876 for more details)

- If neither of them are zero, the fluxes are calculated using the normal HLLC scheme.

\section{Variational data assimilation method}

The variational data assimilation method (also called the 4D-var method) is based on the optimal control theory, [18]. It provides an optimal way of fitting the model to observations by calibrating some model parameters, see e.g. [17, 4] for pioneering articles in which the method is applied to meteorological studies. We apply the method to 2D-SWEs in order to identify hydraulic parameters. The method is based on the minimization of a cost function that measures the discrepancy between the available observations and the computed flow state. The original code (the forward code) has been converted into a more general code which includes the forward model, the adjoint model and an optimization process. The adjoint model is backward in time and includes the observations as source terms. 


\subsection{Adjoint model and optimality condition}

A general cost function $J$ is defined as follows:

$$
J(\mathbf{p})=\int_{0}^{T} \int_{\Omega} \phi\left(\mathbf{U}^{\mathbf{p}}, \mathbf{U}^{o b s}, \mathbf{p}\right) d \Omega d t
$$

where $\phi$ is a smooth functional measuring the discrepancy between the simulated state and the observations and $\mathbf{U}^{\mathbf{p}}$ is the solution of 2D-SWEs (1)-(8). In the trivial case where observations $\mathbf{U}^{o b s}$ are the state variables observed everywhere and at any time, we set:

$$
\phi\left(\mathbf{U}^{\mathbf{p}}, \mathbf{U}^{o b s}, \mathbf{p}\right)=\left\|\mathbf{U}^{o b s}-\mathbf{U}^{\mathbf{p}}\right\|^{2}
$$

Hence $J$ depends on the control variables $\mathbf{p}$ via the state variables $\mathbf{U}^{\mathbf{p}}$. It is an optimal control of $2 \mathrm{D}-\mathrm{SWEs}$. The control variable $\mathbf{p}$ can be the initial condition, the Manning coefficient, the bed elevation, boundary water levels and/or discharge. In the most general case, $\mathbf{p}=\left(\mathbf{U}^{0}, n, Z_{b}, q_{\Gamma}, h_{\Gamma}\right)^{T}$. Of course, in practice one cannot identify every parameter at the same time and $\mathbf{p}$ contains one or only few of these variables.

In the next section, we detail the cost functions we consider. In particular we present some extra penalty terms useful in helping the minimization process converge to better calibrate model.

The cost function is minimized using a local descent algorithm (first order method). Thus, we need to compute the gradient of $J$. We compute it classically by introducing the adjoint state $\mathbf{U}^{*}$ (solution of the adjoint state model) [18]:

$$
\frac{\partial \mathbf{U}^{*}}{\partial t}+\frac{\partial \mathbf{F}^{T}}{\partial \mathbf{U}} \frac{\partial \mathbf{U}^{*}}{\partial x}+\frac{\partial \mathbf{G}^{T}}{\partial \mathbf{U}} \frac{\partial \mathbf{U}^{*}}{\partial y}=-\frac{\partial \mathbf{B}^{T}}{\partial \mathbf{U}} \mathbf{U}^{*}+\frac{\partial \phi}{\partial \mathbf{U}}
$$

where $\mathbf{U}^{*}=\left(h^{*}, q_{x}^{*}, q_{y}^{*}\right)^{T}$ is the adjoint state,

$$
\begin{aligned}
\frac{\partial \mathbf{F}}{\partial \mathbf{U}}^{T}= & \left(\begin{array}{ccc}
0 & -u^{2}+c^{2} & -u v \\
1 & 2 u & v \\
0 & 0 & u
\end{array}\right) \quad \frac{\partial \mathbf{G}}{\partial \mathbf{U}}^{T}=\left(\begin{array}{ccc}
0 & -u v & -v^{2}+c^{2} \\
0 & v & 0 \\
1 & u & 2 v
\end{array}\right) \\
& \frac{\partial \mathbf{B}^{T}}{\partial \mathbf{U}}=\left(\begin{array}{ccc}
0 & g S_{0 x}+\frac{7}{3} g S_{f x} & g S_{0 y}+\frac{7}{3} g S_{f y} \\
0 & -g S_{f x} \frac{2 u^{2}+v^{2}}{u\left(u^{2}+v^{2}\right)} & -g S_{f y} \frac{u}{u^{2}+v^{2}} \\
0 & -g S_{f x} \frac{v}{u^{2}+v^{2}} & -g S_{f y} \frac{u^{2}+2 v^{2}}{v\left(u^{2}+v^{2}\right)}
\end{array}\right)
\end{aligned}
$$

These equations are closed with the final time condition:

$$
\left.\mathbf{U}^{*}\right|_{t=T}=(0,0,0)^{T}
$$

and the following boundary conditions:

- at inflow discharge boundaries and land boundaries,

$$
q_{n}^{*}=0 \text { and } \frac{\partial h^{*}}{\partial x_{n}}=0
$$


- and at water level boundaries,

$$
\left(h^{*}+2 u_{n} q_{n}^{*}\right)=0 \text { and } q_{\tau}^{*}=0
$$

where $\tau$ is the tangential unit vector.

Using the adjoint state, we obtain an expression of the gradient of $J$ independently of $\delta \mathbf{U}$, the derivative of the state with respect to $\mathbf{p}$. The components of the gradient are the following:

- derivative with respect to the initial condition $\mathbf{U}^{0}$

$$
\nabla \mathbf{U}^{0} J=\frac{\partial \phi}{\partial \mathbf{U}^{0}}-\left.\mathbf{U}^{*}\right|_{t=0}
$$

- derivative with respect to the Manning coefficient $n$

$$
\nabla_{n} J=\frac{\partial \phi}{\partial n}-\int_{0}^{T} \frac{\partial \mathbf{B}^{T}}{\partial n} \mathbf{U}^{*} d t
$$

where

$$
\frac{\partial \mathbf{B}^{T}}{\partial n}=\left(0,-\frac{2}{n} g h S_{f x},-\frac{2}{n} g h S_{f y}\right)
$$

- derivative with respect to the bed elevation $Z_{b}$

$$
\nabla z_{b} J=\frac{\partial \phi}{\partial Z_{b}}-\int_{0}^{T} g h\left(\frac{\partial q_{x}^{*}}{\partial x}+\frac{\partial q_{y}^{*}}{\partial y}\right)
$$

- and derivative with respect to the boundary conditions

1. discharge $q_{\Gamma}$

$$
\nabla_{q_{\Gamma}} J=\frac{\partial \phi}{\partial q_{\Gamma}}+h^{*}
$$

2. water depth $h_{\Gamma}$

$$
\nabla h_{\Gamma} J=\frac{\partial \phi}{\partial h_{\Gamma}}+\left(g h-u_{n}^{2}\right) q_{n}^{*}
$$

Generalized model. Finally, we obtain a "generalized model" containing the 2D-SWEs (1) (the forward model), its adjoint model (11) and the necessary optimality condition $\delta J=0$, see e.g. [17]. This generalized model contains the observations since they are integrated into the source term $\phi$ of the adjoint model. The solution of this generalized model provide an optimal fit of the simulated state and the observations; this leads to the calibrated model we seek. 


\subsection{Full optimization process and DassFlow software}

The generalized model described previously is implemented into the software DassFlow, [11]. DassFlow is a software dedicated to variational data assimilation and coupling as applied to river hydraulics. For simulatenous coupling1D2D flows models and data assimilation we refer to [21].

The L-BFGS algorithm [8] is used for minimization. The adjoint solver is not obtained by discretizing the equations (11), using a finite volume scheme, for example, but by directly differentiating the source code, which solves the forward model 2D-SWEs (1). The code differentiation is done using the automatic differentiation tool Tapenade, [10].

The global optimization process can be summarized as follows: given an initial guess of the control variable $\mathbf{p}^{0}$,

1. the forward model 2D-SWEs (1) (finite volume scheme) are solved, and the cost function $J$ is computed - this is the forward code;

2. the adjoint code (obtained by automatic differentiation) is run - this gives the adjoint state and the gradients of $J$;

3. a new control variable value $\mathbf{p}^{k}$ is computed using the iterative minimization algorithm (L-BFGS algorithm, M1QN3 subroutine, [8]);

4. and it is verified that the solution has converged. If the solution has not converged, it is necessary to return to step 1 with the new control variable value $\mathbf{p}^{k}$.

The stop criteria is:

$$
\frac{\left\|\nabla J_{k}\right\|}{\left\|\nabla J_{0}\right\|} \leq \epsilon
$$

where $\left\|\nabla J_{k}\right\|$ and $\left\|\nabla J_{0}\right\|$ are the gradient norm at the initial and current iteration step $k$ respectively, and $\epsilon$ is a small value (typically $10^{-4}$ ).

\section{Test case and cost function}

The efficiency of the proposed method was evaluated through a test case.This test case (described below) contains all the main features of a real case we consider in a forthcoming paper (Moselle River, Part II, see [14]). There are two main reasons to elaborate on our method for a test case:

1. We consider twin experiments. Giving the "unknown" set of parameters, we create the observations numerically. Then, the generated accurate observations are assimilated into model to retrieve this "unknown" set of parameters. Therefore, through twin experiments, it can be easily verified the accuracy of the method. 
Let us add that observations are perfect since they arise from the model. In real cases, models are not perfect and fitting their response with (real) observations generates extra difficulties.

2. A set of observations helps us understand what we can and cannot expect in terms of parameter identification/calibration. Typically, we cannot expect to identify all parameters, boundary conditions and Manning coefficients through the same process. Also, the test case flood duration is much shorter than the real one. Hence, time computations are also much shorter.

\subsection{The test case}

The simulated river is small and consists of a flat main channel (with a bed elevation $-2.5 \mathrm{~m}$, width $10 \mathrm{~m}$ ) and a flood plain with complex topography, see Fig. 1(a). The computational domain is illustrated in Fig.1(b) and discretized with hybrid quadrilateral and triangular grid cells. The mesh consists of 787 cells and 809 nodes.

As mentioned previously, the numerical experiments used synthetic data (twin experiments).

Initial conditions were defined as being the steady flow driven by a constant inflow discharge $Q_{\text {in }}=6.0 \mathrm{~m}^{3} / \mathrm{s}$. This inflow does not lead to flood inundation (the river flows within the main channel). We generate the reference flow by imposing the following inflow discharge (see the reference solution in Fig. 2):

$$
Q_{\text {in }}(t)=6+4.5 t \exp (-t / 60)
$$

\subsection{The cost function}

We assume that observations are elevation values only. These observations come either from gauging stations or satellite images, see e.g. [15, 22]. Those from gauging stations are limited to certain points (two or three different locations at best) but densely distributed over time. On the other hand, if they are extracted from a satellite image they represent only one instant value in time (one image, typically) but are densely distributed over space. We denote the elevation values observed with $h^{o b s}$ and we present below the different terms used to define the cost function we minimize.

\subsubsection{Basic cost function}

Let us assume we have $N$ satellite images with perfect information i.e. observed elevation is available everywhere in the computational domain. The basic cost function is:

$$
J_{o b s}(\mathbf{p})=\frac{1}{2} \sum_{k=1}^{N}\left(\mathbf{h}-\mathbf{h}_{k}^{o b s}\right)^{T} \mathbf{W}^{-1}\left(\mathbf{h}-\mathbf{h}_{k}^{o b s}\right)
$$


where $k$ is the index number of the image and $W$ is a matrix of error covariances which represents the confidence of the quality of observations.

\subsubsection{Extra net-mass flux term}

Only the magnitude of the water level is taken into account in the definition of $J_{o b s}$. However, spatially distributed observations are spread out across space. They can therefore provide information related to the water slope, which is a driving force behind flood flow. Thus, we extend the cost function with an extra penalty term, $J_{\text {flux }}$, which measures the discrepancy of net mass flux (where image is available):

$$
J_{f l u x}(\mathbf{p})=\frac{1}{2} \sum_{k=1}^{N}\left(\mathbf{q}-\tilde{\mathbf{q}}_{k}^{o b s}\right)^{T} \mathbf{W}^{-1}\left(\mathbf{q}-\tilde{\mathbf{q}}_{k}^{\text {obs }}\right)
$$

where $q$ is the computed discharge (net mass flux) and $\tilde{\mathbf{q}}^{\text {obs }}$ is "mix" net mass flux. As a matter of fact, $\tilde{\mathbf{q}}^{\text {obs }}$ is computed using the observed elevation, $h^{o b s}$, and the computed velocity $(u, v)^{T}, \tilde{\mathbf{q}}^{o b s}=h^{o b s}(u, v)^{T}$.

In the next section, we show that this extra term improves the minimization process since it quantifies a discrepancy related to the second component of the state solution $\mathbf{U}$.

\subsubsection{Regularization term and total cost function}

Inverse problems for strongly non-linear systems such as the present one are generally ill-posed, and especially if very few observations are available. In order to regularize the present identification problem (the cost function is a priori non-convex), we introduce the following extra regularization term (Tikhonov regularization $[25])$ :

$$
J_{\text {reg }}(\mathbf{p})=\frac{1}{2}\left|\frac{\partial \mathbf{U}}{\partial t}\right|^{2}
$$

Finally, we minimize the total cost function. $J$ is a weighted sum of $(22),(23)$ and (24):

$$
J=J_{\text {obs }}+\alpha J_{\text {flux }}+\beta J_{\text {reg }}
$$

where $\alpha$ and $\beta$ are weight coefficients.

\subsubsection{Scaling}

A good Hessian matrix condition number is very helpful in further improving the efficiency of the minimization algorithm, particularly if the control variables have different order of magnitude, see e.g. [24]. Here, we use a basic variable transformation method.

We define the scaled control variable as $p^{\prime}=L^{-1} p$, then we obtain the gradient 
$g$ and the Hessian matrix $H$ as follows: $g^{\prime}=L^{T} g$ and $H^{\prime}=L^{T} H L$. Using this transformation, the new components of the control variable $p^{\prime}$ are scaled to unity and the condition number of the new Hessian matrix is improved.

In real problems, difficulties may be encountered in setting up the transformation matrix $L$. For greater simplicity, we choose a diagonal matrix $L$ and set its coefficients such that the different components of the control variable have the same order of magnitude.

\section{Choice of cost function}

In Section 4.2, a cost function, with three terms, to assimilate the spatially distributed water levels image into the flood model is presented. The first term $J_{o b s}$ is a square norm which directly measures the discrepancy between the observed and calculated variables. The third regularization term $J_{r e g}$ improves the ill-posedness of inverse problems, especially the assimilation problem of the spatially distributed water level images which are usually observed at long time intervals. This penalty term is introduced to constrain solutions slowly evolving in time and to obtain the smoothing and physically reasonable solution. Readers may refer to related references for detailed descriptions of the performance of this term, see e.g. [25].

The second term of $J_{\text {flux }}$ is specially proposed to improve the assimilation of the observed image according to the characteristics of the image observations. To examine the performance of this penalty term, the experiment of $Q_{i n}$ identification was conducted by test case. In this experiment, $Q_{i n}$ was considered only control variable and identified with a single full image. The cost function $J$ (Eq. (25)) without a regularization term, i.e. $\beta=0$, is used to understand the special function of $J_{\text {flux }}$ in image assimilation. The first guess was that $Q_{i n}$ is constant, i.e. $Q_{i n}=6.0 \mathrm{~m}^{3} / \mathrm{s}$. Other parameters, such as initial conditions and manning coefficients, were given. The stop criterion $\epsilon$ of the convergence of minimization was set at $10^{-4}$. The maximum number of iteration steps was limited to 50 for contrasting.

Based on this configuration, $Q_{i n}$ was identified with the single observed images taken at different given flooding times: $T_{i m a g}=80 \mathrm{~s}, T_{i m a g}=100 \mathrm{~s}, T_{i m a g}$ $=120 \mathrm{~s}$ and $T_{\text {imag }}=200 \mathrm{~s}$, respectively. In all four experiments, $J_{\text {flux }}$ greatly improved the minimization process, and hence the quality of parameter identification. As an illustration, the identification results with $T_{\text {imag }}=120 \mathrm{~s}$ are plotted in Fig. 2 and Fig. 3. Oscillations in Fig. 2 are due to the lack of regularization (introduced in the term $J_{r e g}$ ). This is a classical behavior of $4 \mathrm{D}$-var algorithms if not using a regularization term. Nevertheless, its lack allows to distinguish the influence of the new term $J_{\text {flux }}$. And it is clearly shown in Fig. 3 that the final value of the observation term $J_{o b s}$ in $J$ is lower than in the one without $J_{f l u x}$. In conclusion, with $J_{\text {flux }}$, one can identify reasonably well $Q_{\text {in }}$ , but without $J_{\text {flux }}$ the convergence of the optimization process is much more difficult and worth. In other words, more information from images is assimilated 
into the flood model by virtue of $J_{\text {flux }}$.

\section{What can we identify with a full image?}

In order to understand the assimilation of spatially distributed water levels, and specifically, to learn, which parameters we can identify from full images (here, a "full image" means that water levels are available in each cell of the computational domain; hereafter, an image is referred as to spatially distributed water level observations), three groups of assimilation experiments were conducted.

\subsection{Identification of initial conditions}

We wanted to identify $\mathbf{U}_{0}$ (initial conditions) only using one single image. Other parameters (inflow discharge, Manning coefficients, etc) were given. The first guess of $\mathbf{U}_{0}$ is still water with water levels interpolated between the upstream and downstream values. Inflow discharge was given by (21) (see the reference solution in Fig. 2).

We considered four cases depending on what time the image was taken: we assimilate one full image at $T_{\text {imag }}=20 \mathrm{~s}, T_{\text {imag }}=40 \mathrm{~s}, T_{\text {imag }}=60 \mathrm{~s}$ (near flood peak time) and $T_{\text {imag }}=80 \mathrm{~s}$, respectively.

The decrease of the cost function vs iterations is plotted in Fig. 4. It is shown that the convergent processes becomes stiff when the observed time of the image is far from the starting time. The assimilation experiments failed due to the numerical instability caused by the over-correction of initial conditions for the image at $T_{\text {imag }}=100 \mathrm{~s}$ (the time at the period of flood recession) or for the subsequent ones.

We computed the root-mean square (RMS) error of the water level $z$ and the norm velocity $\sqrt{u^{2}+v^{2}}$. At a given time $t$ and for a quantity $\phi$ its expression is:

$$
\|\phi\|_{R M S}(t)=\sqrt{\sum \frac{\left(\phi^{i d}(t)-\phi^{r e f}(t)\right)^{2}}{n}}
$$

where, $\phi^{i d}$ and $\phi^{\text {ref }}$ are, respectively, the identified and reference values and $n$ is the number of cells. The RMS errors for water level and velocity norm are plotted in Fig. 5.

Let us recall that we identify the initial conditions. Fig. 5 (a) shows that, after calibration, the lowest error at initial time is for $T_{\text {imag }}=20 \mathrm{~s}$ and the highest one is for $T_{i m a g}=80 \mathrm{~s}$. At final time, the lowest error is, inversely, for $T_{i m a g}=80 \mathrm{~s}$ and the highest for $T_{i m a g}=20 \mathrm{~s}$. All of this remains true for the velocity norm, Fig. 5 (b), except for initial time, where the best RMS error is not obtained for $T_{\text {imag }}=20 \mathrm{~s}$.

As an additional experiment, the 15 images of time interval $t=20$ s during the flood period were assimilated into the model to identify the initial conditions. The corresponding results are also plotted in Fig.4 and 5. The best estimation 
of the flow state throughout the whole flood period is obtained when these observations are assimilated.

The earlier image includes more information from initial conditions than the subsequent one. As flood routing, the initial information may be advected outside of the computational field by the flood wave. The influences of initial conditions on flow states becomes weaker and weaker with the routing of the flood in this limited area. As a matter of fact, one may conclude that the controlled parameters can not be identified unless the assimilated observations include the enough information induced by these parameters. This remark can also be explained by the following experiments.

\subsection{Identification of inflow discharge}

The experiment described in Section 5 was expanded by adding the regularization term $J_{r e g}$, see (25). From this experiment, we wanted to learn whether one can identify an inflow hydrograph with water levels information alone. The configurations of all experiments were the same as those described in Section 5. The results of identified $Q_{i n}$ with single image at $T_{\text {imag }}=80 \mathrm{~s}, T_{\text {imag }}=100 \mathrm{~s}$, $T_{\text {imag }}=120 \mathrm{~s}$ and $T_{\text {imag }}=200 \mathrm{~s}$ respectively, are presented in Fig. 6 . Using the proposed cost function, a smoothing $Q_{i n}$ hydrograph was successfully identified.

For the time of images $T_{i m a g}=80 \mathrm{~s}$ and $T_{i m a g}=100 \mathrm{~s}$, Fig. 6 (a) and (b) respectively, the two cost functions lead to quite similar results. Both allow to identify well the flood peak. However, the extended cost function including the term $J_{\text {flux }}$ gives slightly better results. The cost function without the extra term $J_{\text {flux }}$ fails to identify the flood peak by assimilating images at $T_{\text {imag }}$ $=120 \mathrm{~s}$ and $T_{i m a g}=200 \mathrm{~s}$, Fig. 6 (c) and (d) respectively, while the cost function including all terms gives a good identification of $Q_{i n}$.

From Fig. 2, one may note an interesting but reasonable phenomenon that the inflow discharge has no correction at the time after the observed time. This is because that there are no driven sources (originating from the cost function) for the backward integration of adjoint model when the regularization term in time $J_{r e g}$ is deactivated. Even if the regularization term is active, the hydrograph is smoothed out in time, a significant difference from real conditions.

Physically, the images are observations of the flow state at the observed instant $T_{i m a g}$ which is driven by $Q_{i n}$ before it. Therefore, there are no observations of $Q_{i n}$ after $T_{i m a g}$ to be used to identify the hydrograph.

Thus, one segment of the inflow hydrograph (from 0 to $T_{\text {imag }}$ ) can be successfully identified when assimilating spatially distributed water levels if the proper cost function is adopted. 


\subsection{Identification of initial conditions and inflow discharge}

Finally, we conducted numerical experiments to identify both the inflow discharge and initial conditions. If we observe only a single image as we did previously, the optimization process fails to converge. Observations are not enough to identify both input parameters. If we observe a few full images, we can successfully identify succesfully inflow discharge and initial conditions. We present numerical results obtained from observing 15 full images with time intervals of $20 \mathrm{~s}$, from $t=20 \mathrm{~s}$ to $t=300 \mathrm{~s}$. The identified inflow discharge is shown in Fig. 7(a) and the corresponding cost function and norm gradient which decrease with each iteration step are plotted in Fig.7(b). The differences between the identified and the reference initial conditions in water level and norm velocity, are shown in Fig. 8.

\section{Can we identify the inflow discharge with par- tial images and/or in-situ measurements?}

In real cases, it is not realistic to expect a full dense image like the one considered in the previous section. It is more reasonable to assume that we have at best a single "partial image" and some water level measurements inside the main channel. In this section, we consider numerical experiments utilizing a partial image and partial in-situ measurements with exactly the same configurations as those studied in Part II of this paper (see [14]). We assume that we have the water level hydrograph at a middle gauge station (Fig. 1) but only at the beginning and ending of the flood period $(t \in[0,30] s$ and $t \in[200-300] s)$, see Fig. 9, and potentially one "partial image". By partial image, we mean water levels at the three floodplain blocks, as plotted in Fig. 1(b).

In the legends of subsequent figures, we use "TS" (for "Time Series") to denote results from only the water level hydrograph at the middle gauge station only, and "IMAG" to denote results that take the partial image into account as well.

We conducted three different experiments. In all of them, we observed at least the water level hydrograph at the middle gauge station "TS", and tried only to identify the inflow discharge. Other parameters (initial conditions, Manning coefficients etc) were given. The first guess was constant: $Q_{i n}=6.0 \mathrm{~m}^{3} / \mathrm{s}$.

Let us notice that we treat in detail the identification problem of Manning coefficients in Part II, [14].

Experiment 1. We tried to understand the contribution of one partial image to the identification process. To do so, we did not consider the regularization term $J_{r e g}$ in $(25)$ (i.e. $\beta=0$ ). We performed two identification processes, one with $T_{\text {imag }}=100 \mathrm{~s}$ and $T_{\text {imag }}=120 \mathrm{~s}$ respectively. The corresponding inflow 
hydrograph is plotted in Fig. 10.

First, we note that very large oscillations exist. This is classical and due to the lack of regularization.

The interesting result is that the different images give the same identifiable time interval (see 42s in our experiments, Fig. 10). Let us recall that the the forward model can be viewed as a constraint on the minimization problem. If we've added the regularization term, one would not be able to distinguish precisely the time interval affected by the image. These numerical results illustrate the partial image can constraint the inflow hydrograph successfully during one finite time interval. Also, in the sequel, Experiment 1 is useful to define a good image frequency (see Experiment 3).

Experiment 2. We introduced again the regularization term $J_{\text {reg }}$, from (25), and tried to identify the inflow hydrograph by assimilating the images at $T_{\text {imag }}=100 \mathrm{~s}$ and $T_{\text {imag }}=120 \mathrm{~s}$, respectively. The corresponding results are plotted in Fig. 11.

This experiments also demonstrates that the extra term $J_{\text {flux }}$ from (25) (whether $\alpha$ vanishes or not), is helpful to identify the inflow discharge.

In summary, these numerical experiments show that if the time series ("TS") and partial images are available within the right time interval, it is possible to correctly identify the hydrograph of $Q_{i n}$. Without the extra information provided by the partial images (ie. with TS only), it is impossible to identify $Q_{i n}$.

Experiment 3. Since we noticed in Experiment 1 the need to respect a maximum time interval to correctly identify the flood hydrograph, we used three partial images at $T_{i m a g}=60 \mathrm{~s}, T_{i m a g}=100 \mathrm{~s}$ and $T_{i m a g}=140 \mathrm{~s}$, in this last experiment. The time series was the same as previous experiment. By assimilating these three partial images, it is possible to obtain a perfect identification of $Q_{i n}$. Results, obtained using the complete form of cost function $J$ are plotted in Fig.12.

\section{Overlapping strategy and improvement of com- putational cost}

The adjoint method makes it possible to efficiently compute the gradients of $J$ with respect to control variables. However, the computational cost, including the CPU time and memory requirement, is much higher than that of the classical forward model. The memory requirement, in particular, is enormous. Basically, one needs to store all the state variables $\mathbf{U}$ from the initial time to the final time at each control volume, in order to solve the adjoint model backward in time. Nevertheless, this is not completely true since automatic differentiation tools like Tapenade try now include a strategy for balancing storage and re-computation, see [10]; but the storage approach still remains the basis for adjoint model computation. Thus, the typical amount of memory is insufficient 
for some practical long-duration flood modeling.

[19] propose a temporal strategy that consists of splitting the whole assimilation period into several sub-intervals, then carrying out the assimilation process sequentially over each sub-interval. The final state of the previous sub-interval serve as the first guess of the subsequent sub-interval. [19] focuses on the accuracy of the final time solution for a quasi-geostrophic oceanographic model (the control variable is the initial condition). Then, they defined a "progressive" temporal strategy that better identified for the initial control state.

In the present study, we adapt the sequential temporal strategy of [19] to the specific components of our river hydraulics problem: the identification of inflow hydrograph (time-dependent parameter), Manning coefficients and/or initial conditions (time-independent parameters).

In the following descriptions of the assimilation experiments, we refer the previous sequential strategy as the "temporal strategy without overlapping" and the new strategy defined below as the "temporal strategy with overlapping" .

All the assimilation experiments took the following (dense) observations into account: a water level hydrograph almost continuous over time (elevation values at the gauge station, every $2 \mathrm{~s}$ ) and five partial images at $T_{\text {imag }}=40,60,80,120$ and $160 \mathrm{~s}$.

\subsection{Temporal strategy without overlapping}

The temporal strategy without overlapping efficiently identifies time-independent parameters: from the observations listed above, we identified the Manning roughness coefficients in three different land-use areas (the main channel, left floodplain and right floodplain). We conducted three assimilation experiments: 1) the original experiment which did not include any temporal strategy; 2) the two-sequences experiment which split the assimilation period $[0,300]$ in two subintervals $([0,60] \cup[60,300]) ; 3)$ the four-sequences experiment which split the assimilation period into four sub-intervals $([0,40] \cup[40,80] \cup[80,120] \cup[120,300])$. The first guess of one sub-interval was the converged value of the previous subinterval.

The resulting values are presented in Table 1 . The mean values of the resulting Manning coefficients are close to the true values. The temporal strategy without overlapping works well for the identification of Manning coefficients which are time-independent parameters.

Since there is no overlapping in time and since the speed of convergence of the minimization algorithm is not affected by the time-splitting, the timecomputation of the identification process is similar to that of the process without the temporal strategy. But, the required memory is roughly divided by the number of sub-intervals, much less than that of the original process. 


\subsection{Temporal strategy with overlapping}

\subsubsection{Principles of the algorithm}

We adapted the sequential temporal strategy of [19] (i.e. the temporal strategy without overlapping) to the specific river hydraulics problems. As a matter of fact, the strategy must be adapted to time-dependent parameters like inflow discharge, since information takes time to propagate to the location of observations. Hence, if observations are not dense enough there is a blind period during which the time-dependent parameter is not identifiable. This phenomenon is apparent at the end of the period of assimilation in all of the previously presented figures: the inflow is not well simulated, but rather extended because of the regularization term. In fact, during this period of time, inflow values have no effect on the observations. The blind period represents the time of response of the control variables to the observations. Therefore, we cannot apply the temporal strategy without overlapping to simulate inflow. We propose a new temporal strategy with overlapping. Its fundamental principle is described in Fig. 13 (b).

The assimilation period is divided into several sub-intervals as it was in previous experiments, but with overlapping. We denote the length of this overlapped period with $T_{\text {retro }}$ (retrogressive time). The initial conditions for the new subsequence are defined by the computed flow state of the previous sub-interval. The first guess of control variables in the new sub-sequence is defined as the value identified during the previous sub-interval.

\subsubsection{Identification of inflow discharge}

We present some assimilation experiments based on the temporal strategy with overlapping below. Like the previous experiments, these considered the following observations: a water level hydrograph almost continuous over time (elevation values at the gauge station every $2 \mathrm{~s}$ ) at the gauge station, and five partial images at $T_{\text {imag }}=40,60,80,120$ and 160 s, see Fig. $1 \mathrm{~b}$.

We tried only to identify the inflow discharge $Q_{i n}(t)$. The initial guess was the constant value $Q_{i n}=6 \mathrm{~m}^{3} / \mathrm{s}$. Other parameters were given. We considered four sub-sequences and compared the results obtained using the original method (no temporal strategy) with those obtained using temporal strategy without overlapping and the temporal strategy with overlapping. In the case of no overlapping, the assimilation period was split as follows: $[0,40] \cup[40,80] \cup[80,120] \cup[120,300]$. In the case of overlapping, it was split as follows: $[0,60] \cup[40,100] \cup[80,140] \cup$ $[120,300]$ (i.e. $T_{\text {retro }}=20 \mathrm{~s}$ ). The resulting inflow discharge is plotted in Fig.14.

The original method and that based on the temporal strategy with overlapping give excellent results: we retrieved the reference solution. The temporal strategy without overlapping, on the other hand, does not provide a very good 
solution. Because it enables us to identify $Q_{\text {in }}(t)$ while shortening the assimilation periods, hence saving memory. The temporal strategy with overlapping, is perhaps the most preferable method.

Since overlapping time periods are introduced in the new strategy, its computational time is slightly longer than that of the original one. Nevertheless, these experiments show that the extra computational time is negligible. We present computational times of the present experiments in Table 2. The total time remains almost the same with or without the temporal strategy since the convergence of the minimization process is slighltly improved when using the temporal strategy with overlapping.

\section{Conclusion}

Using a variational data assimilation approach (4D-var), we have investigated potential contributions of spatially distributed water level data to the identification of both time-independent parameters (initial condition, Manning coefficients) and time-dependent parameters (inflow discharge) in a shallow-water flood model. In real-world flooding situation such as the one addressed in Part II [14], spatially distributed water level data are extracted from a satellite image. Such images offer spatially distributed information of the flood stage in the floodplain at one time instant, while more classical measurements, such as water level values at a gauge station offer data points distributed across time.

Numerical experiments conducted in this study indicate that data distributed densely over space is of great benefit to the identification of unknowns parameters (initial conditions and/or inflow). However, accurate identification of timedependent parameters (e.g. inflow) depends greatly on the time of the available image. They have been successfully identified when both a partial image and partial in situ mesurements (water levels) are available. As an example, we have demonstrated the simulation of inflow and reconstruction the flood flow state. This shows that water level observation images may compensate for unavailable in situ measurements during a flood event.

We have contributed to the evolving methodology by introducing an extra term into the cost function that greatly improves the identification process. This term provides some information related to the velocity components of the model, despite the fact that only observations of water level are assimilated. We have also introduced a new temporal strategy with overlapping that can remedy the memory requirement difficulties of long-term simulations. This temporal strategy achieves a prediction of the same quality as the original data assimilation process but with much less memory and with similar CPU time.

This study contributes to the basic understanding of the assimilation of spatially distributed water levels into shallow-water flood models. It demonstrates that the use of a satellite image improves parameter identification processes, 
and, thus, flood prediction. However, a more challenging task is the assimilation of actual remote sensing data with many unknown (and uncontrolled) errors. The real application of the proposed method is demonstrated in a forthcoming paper (Part II).

\section{Acknowledgements}

The authors would like to thank Christian PUECH (Cemagref Montpellier, France) for his very important help to setting up the problem.

This research has been supported by Région Rhône-Alpes, France ( "numerical prevention for floods" project 2003-2006). The authors would like to thank the reviewers for their constructive comments.

English writing has been greatly improved thanks to National Natural Science Foundation of China (Grant No. 50709034).

\section{References}

[1] G.A. Atanov, E.G. Evseeva, and E.A. Meselhe. Estimation of roughness profile in trapezoidal open channels. Journal of Hydraulic Engineering, 125(3):309-312, 1999.

[2] E. Belanger and A. Vincent. Data assimilation (4D-VAR) to forecast flood in shallow-waters with sediment erosion. Journal of Hydrology, 300:114$125,2005$.

[3] W. Castaings, D. Dartus, M. Honnorat, F-.X. Le Dimet, Y. Loukili, and J. Monnier. Automatic differenciation: a tool for variational data assimilation and adjoint sensitivity analysis for flood modeling. Lecture Notes in Computational Science and Engineering, 50:249-262, 2006.

[4] P. Courtier and O. Talagrand. Variational assimilation of meteorological observations with the direct adjoint shallow water equations. Tellus, 42(A):531-549, 1990.

[5] Y. Ding, Y. Jia, and S.Y. Wang. Identification of Manning's roughness coefficients in shallow water flows. Journal of Hydraulic Engineering, 130(6):501-510, 2004.

[6] E. Fernández-Nieto, D. Bresch, and J. Monnier. A consistent intermediate wave speed for a well-balanced hllc solver. C. R. Acad. Sci. Paris, Ser. I, 346:795-800, 2008.

[7] I. Gejadze and J. Monnier. On a 2d zoom for 1d shallow-water model: coupling and data assimilation. Comp. Meth. Appl. Mech. Eng., 196(4548):4628-4643, 2007. 
[8] J.C. Gilbert and C. Lemaréchal. Some numerical experiments with variablestorage quasi-newton algorithms. Mathematical Programming, 45(1):407435, 1989.

[9] J. Hartnack and H. Madsen. Data assimilation in river flow modelling. In $4^{\text {th }}$ DHI software conference, 6-8 june 2001, Scanticon Conference Center, Helsing $\varnothing \mathrm{r}$, Danemark, 2001.

[10] L. Hascoet and V. Pascual. Tapenade 2.1 user's guide. Technical Report RT-300, INRIA, project Tropics, www-sop.inria.fr/tropics, 2004.

[11] M. Honnorat, J. Marin, J. Monnier, and X. Lai. Dassflow v1.0: a variational data assimilation software for river flows. Research report INRIA RR-6150, 2007.

[12] M. Honnorat, J. Monnier, and FX. Le Dimet. Lagrangian data assimilation for river hydraulics simulations. Comput. Visual. Sc., Published on-line, march 2008.

[13] M. Honnorat, J. Monnier, N. Rivière, E. Huot, and FX. Le Dimet. Identification of equivalent topography in an open channel flow using lagrangian data assimilation. Comput. Visual. Sc., Accepted, to appear, 2009.

[14] R. Hostache, X. Lai, J. Monnier, and C. Puech. Assimilation of spatial distributed water levels into a shallow-water flood model. part ii: use of a remote-sensing image of mosel river. J. Hydrology, To appear.

[15] R. Hostache, C. Puech, G. Schumann, and P. Matgen. Estimation de niveaux d'eau en plaine inondée à partir d'images satellites radar et de données topographiques fines. Revue Télédétection, 6(4):325-343, 2006.

[16] N.M. Hunter, P.D. Bates, M.S. Horritt, A.P.J. De Roo, and M. G. F. Werner. Utility of different data types for calibrating flood inundation models within a glue framework. Hydrol. Earth Syst. Sci., 9:412-430, 2005.

[17] F.X. Le Dimet and O. Talagrand. Variational algorithms for analysis assimilation of meteorological observations: theoretical aspects. Tellus A, 38:97-110, 1986.

[18] J.L. Lions. Optimal control of systems governed by partial differential equations. Springer-Verlag, 1971.

[19] B. Luong, J. Blum, and J. Verron. A variational method for the resolution of a data assimilation problem in oceanography. Inverse Problems, 14(4):979$997,1998$.

[20] H. Madsen, J. Hartnack, and J. V. Sørensen. Data assimilation in a flood modelling system using the Ensemble Kalman filter. In $C M W R-\mathrm{XvI}$, Copenhaguen, Danemark, june 2006. 
[21] J. Marin and J. Monnier. Superposition of local zoom model and simultaneous calibration for floodplain flows. Math. Comput. Simul., To appear.

[22] C. Puech, R. Hostache, D. Raclot, and Matgen P. Estimation of flood water levels by merging dem and satellite imagery using hydraulics laws through ai to enhances the estimates. Proceedings of Second Space for Hydrology Workshop. ESA. Geneva, nov. 2007.

[23] H. Roux and D. Dartus. Parameter identification using optimization techniques in open-channel inverse problems. J. Hydraul. Res., 43:311-320, 2005 .

[24] W.C. Thacker. The role of the hessian matrix in fitting models to measurements. Journal of Geophysical Research, 94(C5):6177-6196, 1989.

[25] A.N. Tikhonov and V.Y. Arsenin. Solution of Ill-Posed Problems. Winston, Washington, DC, 258, 1977.

[26] E.F. Toro. Shock-capturing methods for free-surface shallow flows. J. Wiley, New York, 2001.

[27] DH Zhao, HW Shen, GQ Tabios, JS Lai, and WY Tan. Finite-Volume TwoDimensional Unsteady-Flow Model for River Basins. Journal of Hydraulic Engineering, 120(7):863-883, 1994. 


\section{List of Figures}

1 Test case. (a): topography; (b): mesh, gauge station and partial observation areas (image areas). . . . . . . . . . . . . . 23

2 Identification of $Q_{i n}$ with a single full image at $T_{\text {imag }}=120 \mathrm{~s}$. Comparison of identified discharge when minimizing $J_{o b s}$ (solid line) and $J_{o b s}+J_{\text {flux }}$ (long dashed line). The circles are the reference solution. $Q_{\text {in }}$ cannot be identified after the observed time (see detailed explanation in the text). . . . . . . . . 24

3 Identification of $Q_{i n}$ with a single full image at $T_{\text {imag }}=120 \mathrm{~s}$. Cost function decreasing with iteration steps. Basic cost function in red solid line; new cost function in green dashed-line. . . . . . 25

4 Identification of initial conditions with single full image: cost function vs iterations . . . . . . . . . . . . . . 25

5 Identification of initial conditions with single full image: RMS errors of water level (a) and norm velocity (b) . . . . . . 26

6 Identification of $Q_{i n}$ with one single full image at $T_{\text {imag }}=80 \mathrm{~s}$ (a) or $T_{i m a g}=100 \mathrm{~s}(\mathrm{~b})$ or $T_{\text {imag }}=120 \mathrm{~s}$ (c) or $T_{\text {imag }}=200 \mathrm{~s}$ (d): Comparison of the identified discharge by minimizing $J_{o b s}$ (solid line) or $J_{o b s}+J_{\text {flux }}$ (long dashed line). . . . . . . . . . 27

7 Identification of initial conditions and inflow discharge by assimilating 15 full images: comparison of identified discharge with (solid line) and without (long dashed line) regularization (a) and convergence process with regularization $(\mathrm{b}) \ldots \ldots \ldots \ldots$

8 Identification of initial conditions and inflow discharge by assimilating 15 full images: differences between identified and true initial conditions in water level $(Z)\left(\right.$ a) and norm velocity $\sqrt{u^{2}+v^{2}}$

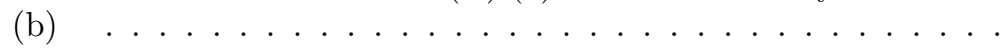

9 Experiments with partial images and in-situ measurements: observations available in time. . . . . . . . . . . . . . . 29

10 Experiments with a partial image and in-situ measurements "TS": Comparison of identified $Q_{i n}$ with "TS" only (solid line), 'TS' and 'IMAG' at $T_{\text {imag }}=100$ s (long dashed line), 'TS' and 'IMAG' at $T_{i m a g}=120 \mathrm{~s}$ (short dashed line) using the cost function without

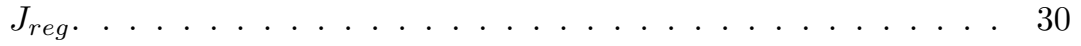

11 Experiments with a partial image and in-situ measurements "TS": Comparisons of identified discharge with image at $T_{\text {imag }}=100 \mathrm{~s}$ (a) and $T_{\text {imag }}=120 \mathrm{~s}$ (b), using cost function with or without the extra term $J_{f l u x} \ldots \ldots \ldots \ldots \ldots \ldots \ldots$

12 Experiments with three partial images and measurements in-situ "TS": Identification of inflow discharge (dot line). . . . . . . . 31

13 Temporal strategy: a) without overlapping; b): with overlapping (with retrogressive time $T_{\text {retro }}$ ) $\ldots \ldots \ldots$. . . . . . . 31 
14 Identification of inflow discharge and temporal strategies: comparison of the original method (solid line), temporal strategy without overlapping (long dashed line) and temporal strategy with overlapping (short dashed line) . . . . . . . . . . 31

\section{List of Tables}

1 Temporal strategy: Comparison of identified and true values of $n \quad 24$

2 Temporal strategy experiments: computational costs . . . . . 26 


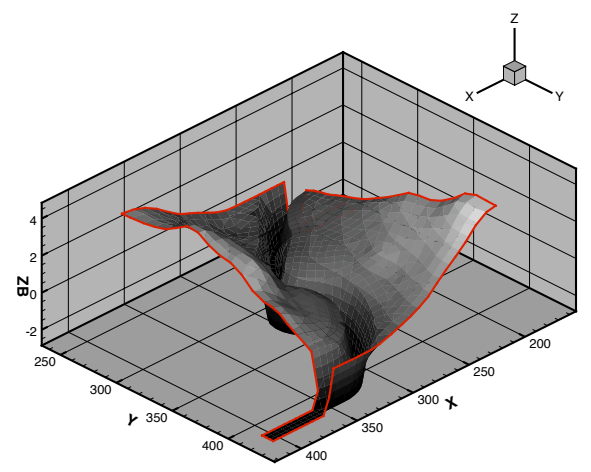

(a)

(b)

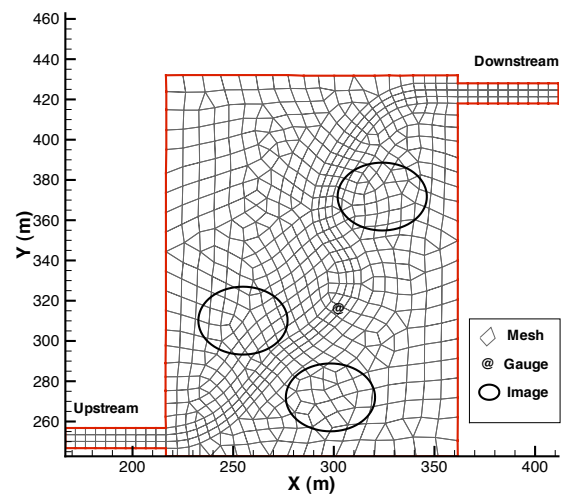

Figure 1: Test case. (a): topography; (b): mesh, gauge station and partial observation areas (image areas). 


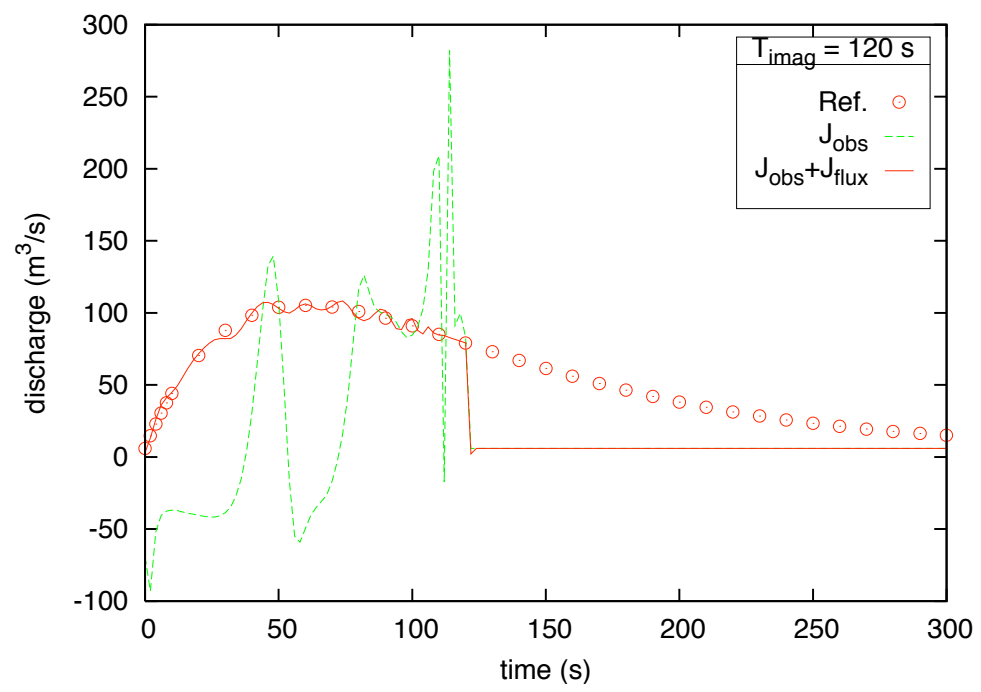

Figure 2: Identification of $Q_{i n}$ with a single full image at $T_{i m a g}=120 \mathrm{~s}$. Comparison of identified discharge when minimizing $J_{o b s}$ (solid line) and $J_{o b s}+J_{\text {flux }}$ (long dashed line). The circles are the reference solution. $Q_{i n}$ cannot be identified after the observed time (see detailed explanation in the text).

Table 1: Temporal strategy: Comparison of identified and true values of $n$

\begin{tabular}{llrrr}
\hline Experiment & Sequence & Main channel & Left floodplain & Right floodplain \\
\hline & True value & 0.0180 & 0.030 & 0.040 \\
& First Guess & 0.0100 & 0.0100 & 0.0100 \\
\hline Original & $0-300 \mathrm{~s}$ & 0.0181 & 0.0313 & 0.0404 \\
\hline Two Seq. & $10-60 \mathrm{~s}$ & 0.0200 & 0.0316 & 0.0402 \\
& $260-300 \mathrm{~s}$ & 0.0178 & 0.0314 & 0.0411 \\
& Mean value & 0.0187 & 0.0315 & 0.0407 \\
\hline Four Seq. & $10-40 \mathrm{~s}$ & 0.0195 & 0.0318 & 0.0399 \\
& $240-80 \mathrm{~s}$ & 0.0191 & 0.0304 & 0.0404 \\
& $380-120 \mathrm{~s}$ & 0.0189 & 0.0299 & 0.0402 \\
& $4120-300 \mathrm{~s}$ & 0.0174 & 0.0329 & 0.0370 \\
& Mean value & 0.0187 & 0.0313 & 0.0394 \\
\hline
\end{tabular}




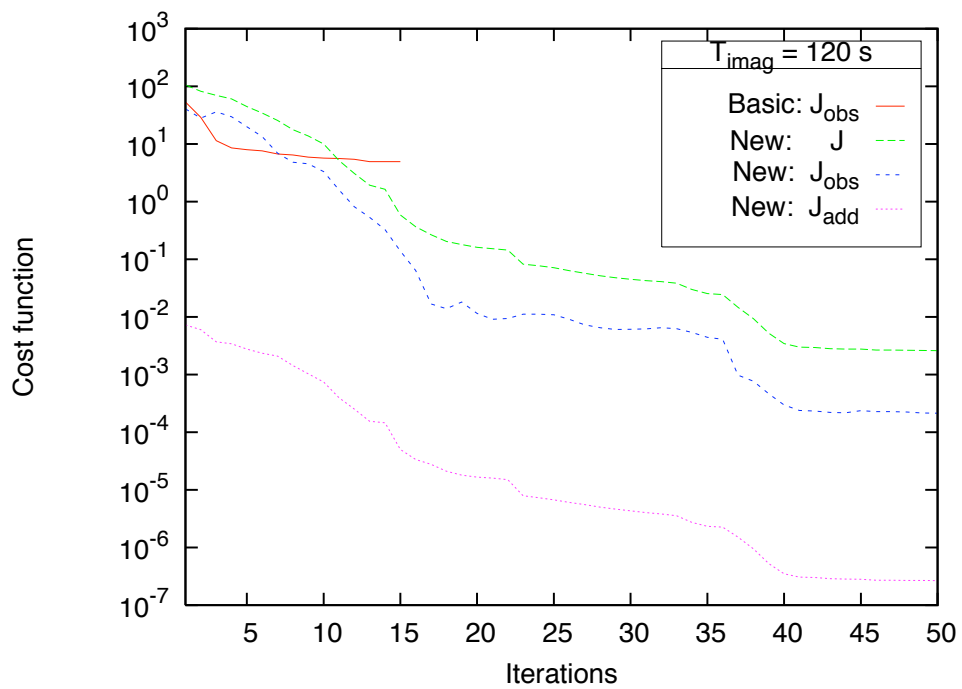

Figure 3: Identification of $Q_{i n}$ with a single full image at $T_{i m a g}=120 \mathrm{~s}$. Cost function decreasing with iteration steps. Basic cost function in red solid line; new cost function in green dashed-line.

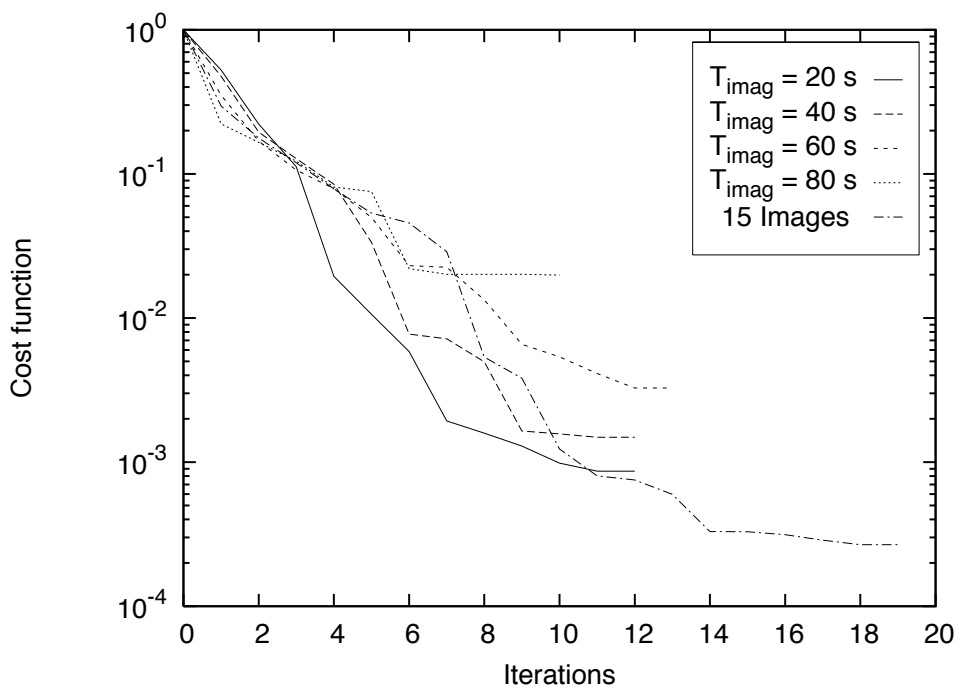

Figure 4: Identification of initial conditions with single full image: cost function vs iterations 


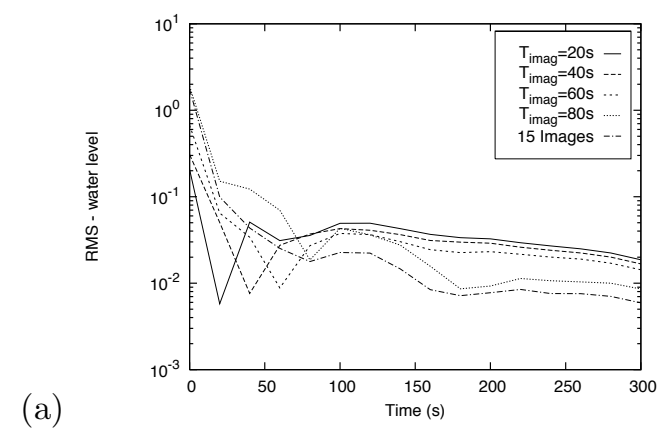

(b)

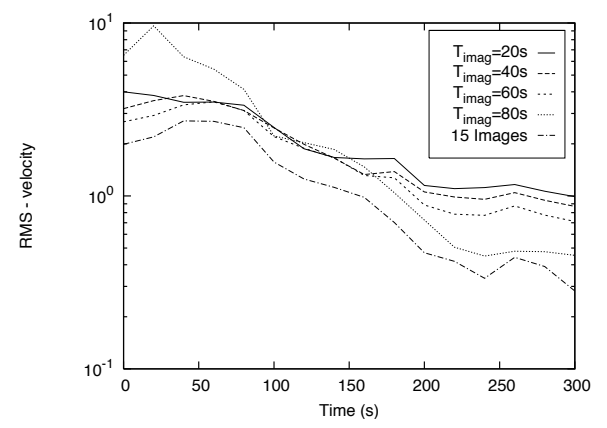

Figure 5: Identification of initial conditions with single full image: RMS errors of water level (a) and norm velocity (b)

Table 2: Temporal strategy experiments: computational costs

\begin{tabular}{lcr}
\hline Experiment & Number of Sequences & CPU time $(\mathrm{s})$ \\
\hline Original & 1 & 84.990 \\
$T_{\text {retro }}=0 \mathrm{~s}$ & 4 & 67.030 \\
$T_{\text {retro }}=20 \mathrm{~s}$ & 4 & 85.071 \\
$T_{\text {retro }}=25 \mathrm{~s}$ & 4 & 86.074 \\
$T_{\text {retro }}=30 \mathrm{~s}$ & 4 & 110.598 \\
\hline
\end{tabular}




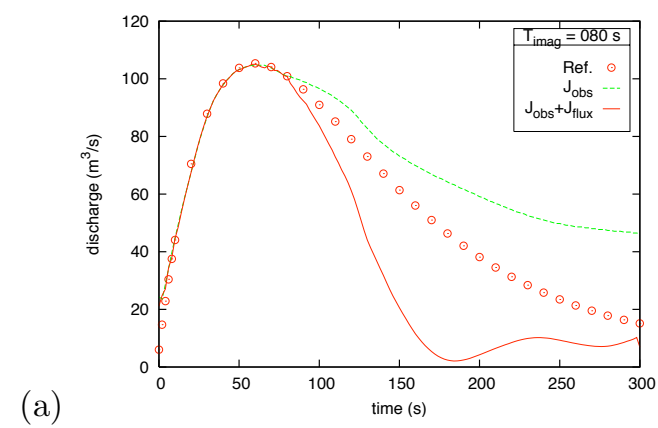

(b)

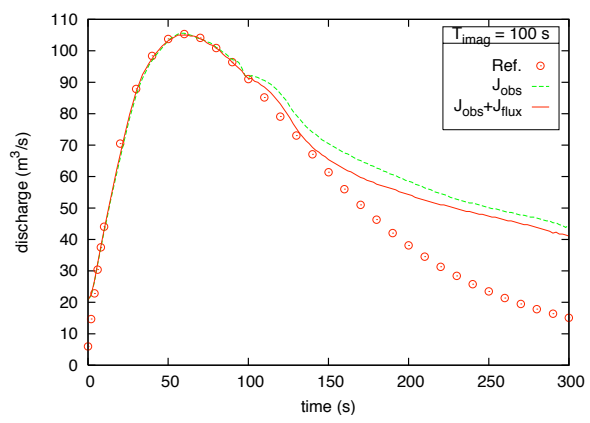

(c)

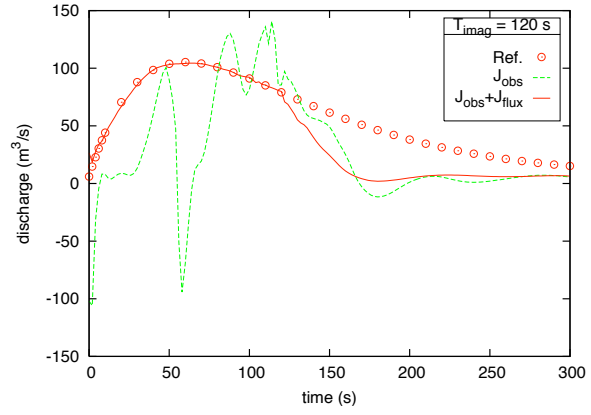

(d)

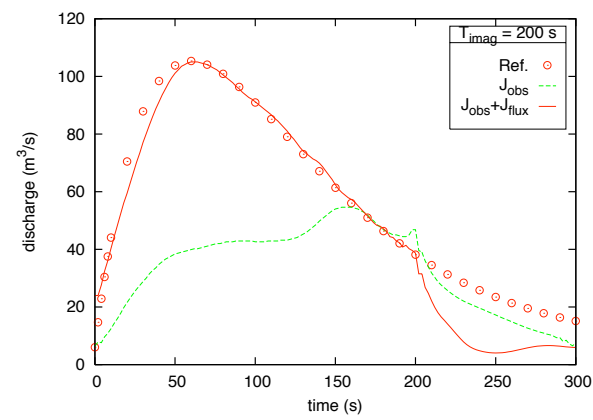

Figure 6: Identification of $Q_{i n}$ with one single full image at $T_{\text {imag }}=80 \mathrm{~s}$ (a) or $T_{\text {imag }}=100 \mathrm{~s}(\mathrm{~b})$ or $T_{\text {imag }}=120 \mathrm{~s}$ (c) or $T_{\text {imag }}=200 \mathrm{~s}$ (d): Comparison of the identified discharge by minimizing $J_{o b s}$ (solid line) or $J_{o b s}+J_{\text {flux }}$ (long dashed line). 

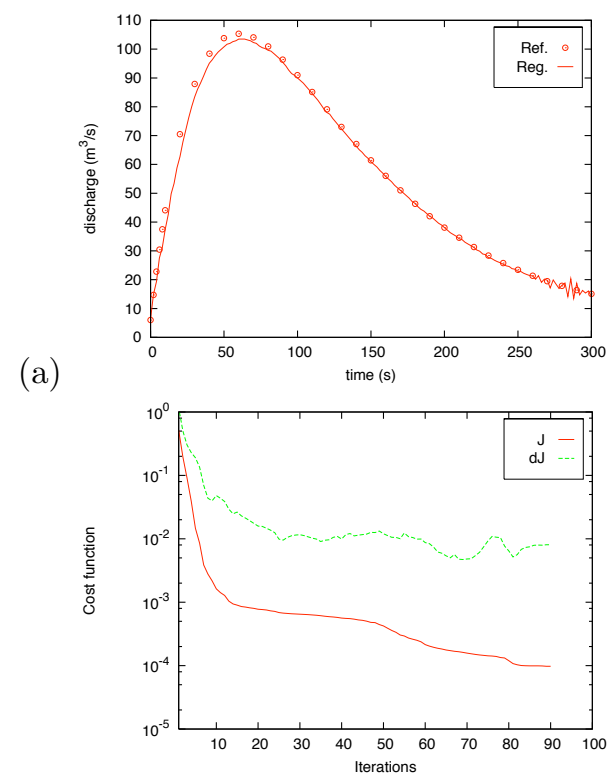

Figure 7: Identification of initial conditions and inflow discharge by assimilating 15 full images: comparison of identified discharge with (solid line) and without (long dashed line) regularization (a) and convergence process with regularization (b) 


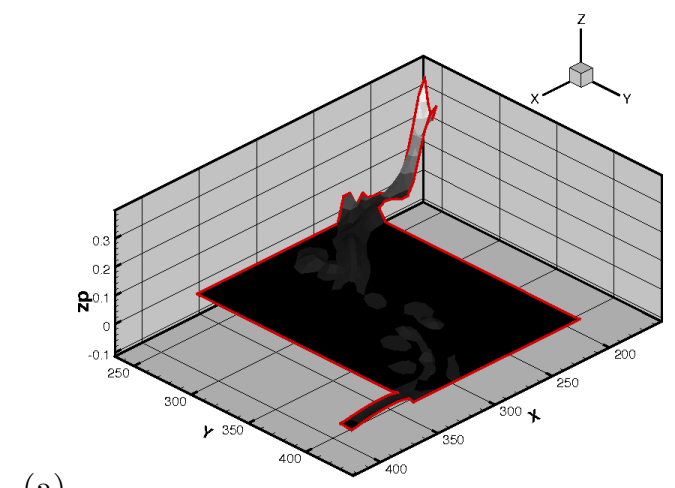

(a)

(b)

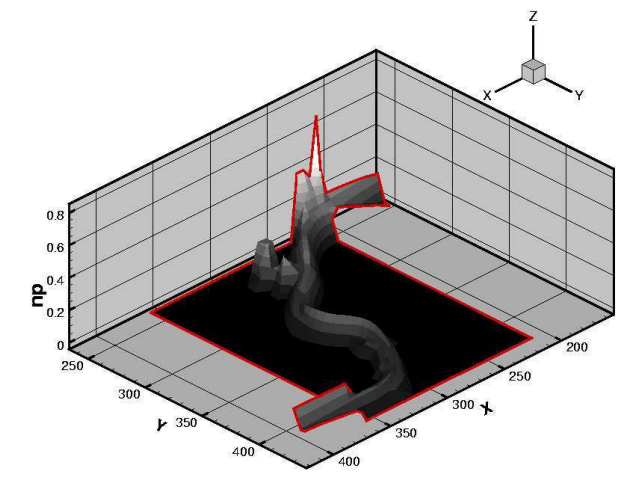

Figure 8: Identification of initial conditions and inflow discharge by assimilating 15 full images: differences between identified and true initial conditions in water level $(Z)$ (a) and norm velocity $\sqrt{u^{2}+v^{2}}$ (b)

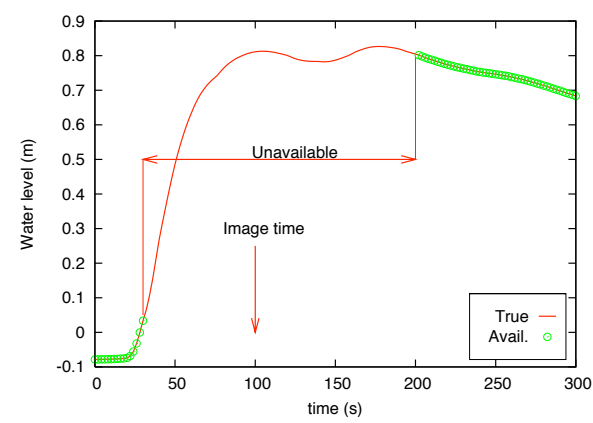

Figure 9: Experiments with partial images and in-situ measurements: observations available in time. 


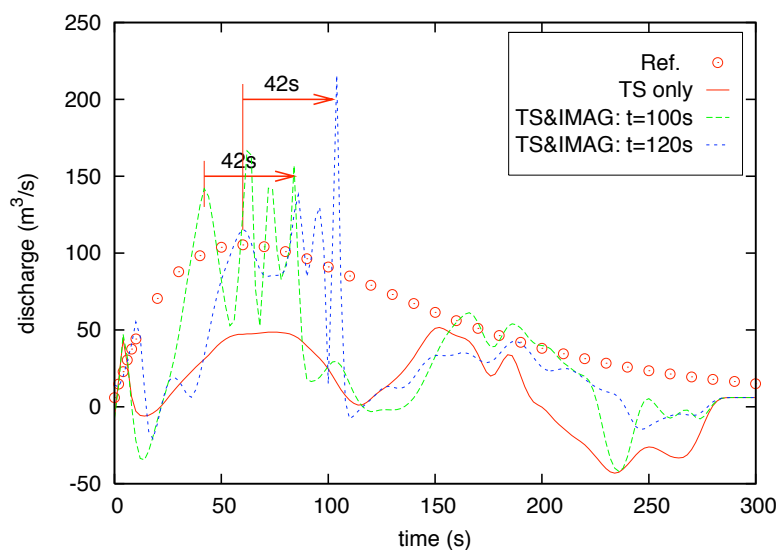

Figure 10: Experiments with a partial image and in-situ measurements "TS": Comparison of identified $Q_{\text {in }}$ with "TS" only (solid line), 'TS' and 'IMAG' at $T_{\text {imag }}=100 \mathrm{~s}$ (long dashed line), 'TS' and 'IMAG' at $T_{i m a g}=120 \mathrm{~s}$ (short dashed line) using the cost function without $J_{r e g}$.

(a)

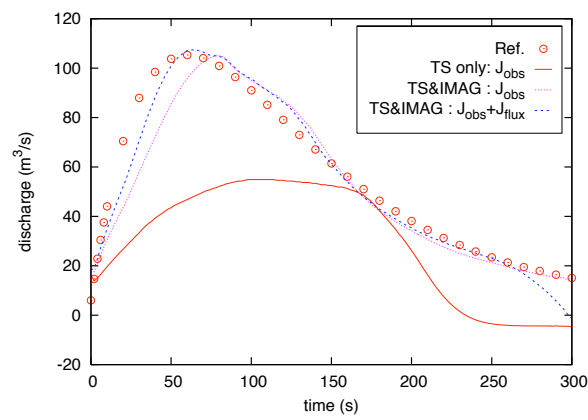

(b)

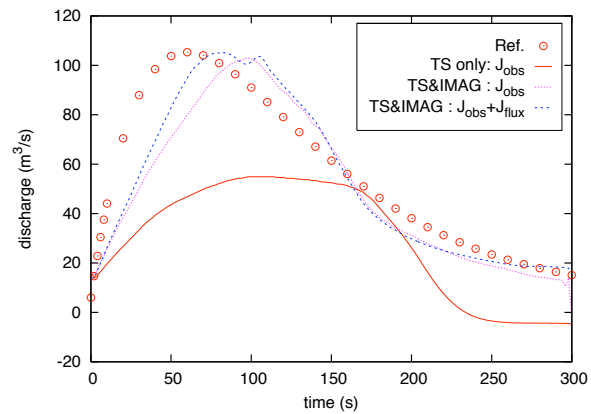

Figure 11: Experiments with a partial image and in-situ measurements "TS": Comparisons of identified discharge with image at $T_{\text {imag }}=100 \mathrm{~s}$ (a) and $T_{i m a g}=$ $120 \mathrm{~s}$ (b), using cost function with or without the extra term $J_{\text {flux }}$ 


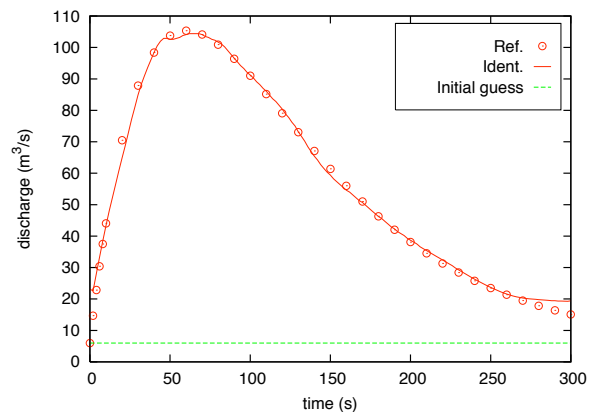

Figure 12: Experiments with three partial images and measurements in-situ "TS": Identification of inflow discharge (dot line).

(a)

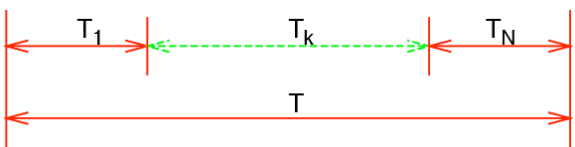

(b)

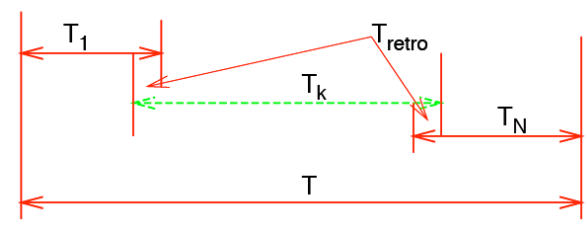

Figure 13: Temporal strategy: a) without overlapping; b): with overlapping (with retrogressive time $T_{\text {retro }}$ )

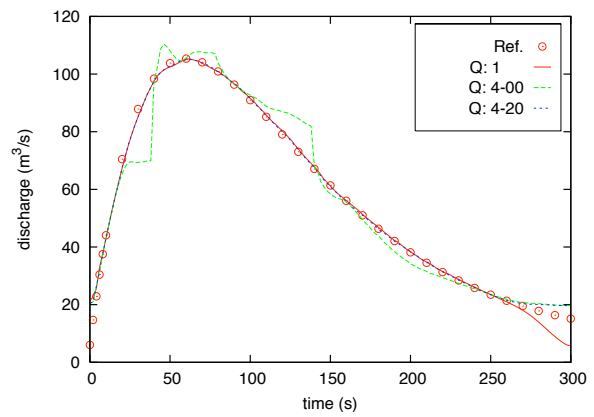

Figure 14: Identification of inflow discharge and temporal strategies: comparison of the original method (solid line), temporal strategy without overlapping (long dashed line) and temporal strategy with overlapping (short dashed line). 